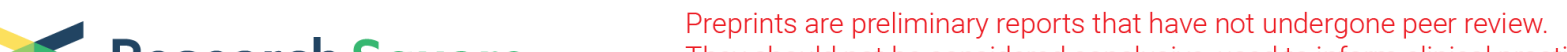 Research Square
They should not be considered conclusive, used to inform clinical practice,
or referenced by the media as validated information.
}

\section{Authorized, Clear and Timely Communication of Risk to Guide Public Perception and Action: Lessons of COVID-19 from China}

Ni Gong

Jinan University

Jing Liao ( $\square$ liaojing5@mail.sysu.edu.cn )

School of Public Health, Sun Yat- sen University

Xiaoyuan Jin

University of Minnesota Twin Cities: University of Minnesota https://orcid.org/0000-0003-0937-3738

Yundong Li

Guangdong Academy of Social Sciences

Meifen Zhang

Sun Yat-Sen University

Yu Cheng

Sun Yat-Sen University

Dong Xu

Sun Yat-Sen University

\section{Research article}

Keywords: COVID-19, Information, Risk perception, Prevent measures, Risk communication, Lessons from China

Posted Date: February 9th, 2021

DOI: https://doi.org/10.21203/rs.3.rs-192109/v1

License: (c) (1) This work is licensed under a Creative Commons Attribution 4.0 International License.

Read Full License

Version of Record: A version of this preprint was published at BMC Public Health on August 12th, 2021. See the published version at https://doi.org/10.1186/s12889-021-11103-1. 


\section{Abstract}

Backgrounds: This study examined the dynamic association between risk communication and public's risk perception and action across the COVID-19 outbreak timeline in China.

Methods: Publicly available information on COVID-19 was collected by Parehub tool for official channels (e.g. government websites and official media), and by Zhongyun Big Data Platform for mainstream Chinese social media (e.g. TikTok, Weibo, etc.). Information was examined by its release channels, content and release time. An online survey was conducted via WeChat across 33 provinces and municipalities of China to assess changes in participants' risk perception and action against COVID-19. Information content and release-time trajectories were examined against the public's risk perception and actions over time.

Results: Altogether, 1,477 pieces of authorized information and 297,000 pieces of short videos on COVID19 were retrieved from official channels and social media. Our online survey of 1,311 responds (25-60 years, $42 \%$ male) indicated that participants mainly relied on official channels to obtain information. Alongside the progress of the outbreak, there was a gradual rise in information quantity, publishing frequency, and content variation. Correspondingly, the public's risk perception that "take it seriously" rose from $13 \%$ to $80 \%, 87.1 \%$ of who took "multiple actions" compared to $25.9 \%$ initially.

Conclusions: Our findings indicated that insufficient information freely-accessible at the early stages of the outbreak may nevertheless lead to the lack of risk awareness and inadequate protective actions of the public. Given the current global situation of COVID-19, the study highlights authorized, transparent, and timely two-way risk communication is vital to guide public perception and actions.

\section{Introduction}

The first case of pneumonia caused by a novel coronavirus was reported to the World Health Organization (WHO) from Wuhan (Hubei province) in China on 31 December 2019 [1]. On 11 March 2020, WHO officially declared the 2019 coronavirus epidemic (COVID-19) as a pandemic [1]. As a response to the pandemic, countries worldwide have been taking measures in preventing and controlling the pandemic in hopes of slowing the spread-governments imposed travel bans on an unprecedented scale to contain the transmission, closing their borders and implementing mandatory screening of citizens returning from heavily affected areas [2, 3]. Despite these measures, the numbers of COVID-19 cases and deaths continue to rise globally [2, 3]. Follow with reported daily new incidence cases from WHO, after 7 March 2020, the new incidence cases from foreign countries grow rapidly, even the increased trend was hard to control that growth repeatedly when has the decreased cases. However, Fig. 1 also expressed, as the first country impacted by COVID-19, China has had steady declines in the number of new cases since March, and confirmed internal cases are controlled, even close to zero [1, 7]. This achievement, as highlighted by the WHO, is not possible without the collective willpower of the public [8]. We examined the dynamic relationship between publicly- available information by its release channels, content and publish 
time, and the Chinese public's risk perception and action across the outbreak timeline; aiming to provide the global community with firsthand evidence of effective risk communication to guide public risk perception and self- protection.

\section{Methods}

Information on COVID-19 publicly- available was mainly released online through official channels (e.g. government websites and official media) and social media (e.g. TikTok, Weibo, etc.) in China [9]. In view of recent studies on social media's effects on risk communication $[11,12]$, we combined social media data from given publications via a scoping review method and these captured by Zhongyun Big Data Platform[10]. Information released by official channels was collected by the Parehub tool. We defined official channels as national and local health commissions websites, People's Daily Online News and CCTV news; and set the examination period from 8 December 2019 (the first reported pneumonia case) and 10 February 2020 (after the Chinese Lunar New Year). Using key terms "new coronavirus", "epidemic", and "pneumonia", we extracted information related to disease (epidemic profile of the outbreak), preventive behaviors (preventative and measurements guidance), and social welfare (logistical and transportation support, etc.). By matching these words from JavaScript and AJAX pages, we collected data in seconds. These data were all free-accessible, and thus did not require ethics approval.

To access the public reaction toward to COVID-19 over the same time period, we conducted an online survey via the Wenjuanxing platform (https://www.wjx.cn/app/survey.aspx). Because of the sudden outbreak of COVID-19, the study designed the questionnaire by reviewing a large amount of relevant literatures related to SARS. Further, the questionnaire is finalized by consulting relevant experts for continuous improvement and modification. Participants were recruited through a snowball sampling method. All of participants have read and signed the informed context before starting the survey. The survey repeatedly assessed participants' trust in different sources of information, risk perceptions and prevent measures in four specific time periods denoted by significant events of the outbreak or two National Holidays (Fig. 2, line chart). Participants read and signed informed consent before participation. This study was approved by the Institutional Review Board (IRB) of the Anthropology Department at Sun Yat-sen University.

We calculated the number of publications to estimate the changes in trends. We also evaluated the percentage of the public's perceived risk and measures taken versus their answered choices. Specifically, through comparing the percentage differences of each parameter under different periods, we were able to observe their changes in trends. Finally, we compared the changes in trends to that of published information, level of perceived risk and taken measures to observe the effects among them.

\section{Study Results}

Altogether, 1477 pieces of authorized information on COVID-19 were retrieved from the national and local health commissions websites $(n=180,12 \%)$, People's Daily Online News $(n=806,55 \%)$ and CCTV news 
( $n=491,33 \%$ ) via the Parsehub tool. Over the same period, 297,000 pieces of short videos were found using the keyword "new coronavirus" and "suspected pneumonia," which were released by mainstream Chinese social media and had been played over 21.8 billion times [9]. These channels were also selected as primary information sources by the 1311 respondents of our online survey, who were between the age of $25-60$ years. $42 \%$ of these participants were males, and $68 \%$ had a bachelor degree or above (as shown in Table 1).

\section{Table 1. Recruited Participants' Demographical Information}

\begin{tabular}{|c|c|}
\hline & Patients $(n=1311)$ \\
\hline \multicolumn{2}{|l|}{ Sex } \\
\hline Male & $554(42.3 \%)$ \\
\hline Female & $757(57.7 \%)$ \\
\hline \multicolumn{2}{|l|}{ Age } \\
\hline$\leqslant 25$ & $258(19.7 \%)$ \\
\hline $26-35$ & $437(33.3 \%)$ \\
\hline $36-45$ & $282(21.5 \%)$ \\
\hline $46-60$ & $307(23.4 \%)$ \\
\hline$>60$ & $27(2.0 \%)$ \\
\hline \multicolumn{2}{|l|}{ Degree } \\
\hline High school/secondary school and below & $185(14.1 \%)$ \\
\hline Junior college & $237(18.1 \%)$ \\
\hline Bachelor & $655(50.0 \%)$ \\
\hline Master's degree and above & $234(17.9 \%)$ \\
\hline \multicolumn{2}{|l|}{ Income (yuan) } \\
\hline$\geqslant 4000$ & $199(15.2 \%)$ \\
\hline $4001-8000$ & $476(36.3 \%)$ \\
\hline$<8000$ & $636(48.5 \%)$ \\
\hline \multicolumn{2}{|l|}{ Family size (number of family member) } \\
\hline$\leqslant 2$ & $114(8.7 \%)$ \\
\hline $3-5$ & $1033(78.8 \%)$ \\
\hline$>5$ & $164(12.5 \%)$ \\
\hline \multicolumn{2}{|l|}{ Marriage status } \\
\hline Unmarned & $443(33.8 \%)$ \\
\hline Married & $827(63.1 \%)$ \\
\hline Divorced & $35(2.7 \%)$ \\
\hline Widowed & $6(0.5 w)$ \\
\hline
\end{tabular}

The survey results show that only $24 \%$ of responses chose to "obtain epidemic information" through TikTok, and only $4.8 \%$ believed that transmitted high credibility information. On the other hand, $85.76 \%$ of people choose to "obtain epidemic information" through "official news," and $58.8 \%$ of participants tend to obtain the disease data through the "National Health Commission, Centers for Disease Control, and the official websites of hospitals." Among them, $88.9 \%$ of the masses believe "official news" as a channel for 
obtaining highly credible information. Despite the high trust in authorized information released by official channels, the contrasting amount of information released by official channels versus social media heightens the possibility of information overload to the public.

We further analyzed the content and release time of authorized information by official channels against the public's perceived risk and actions. During the window period, as a kind of special and new disease, the public's knowledge about COVID-19 is low. These had less information publicly available to announce and promote the disease, so only two disease-related announcements were released by the Wuhan Municipal Health Commission. The rising period was during Chunyun, the World's largest annual human migration. A gradual rise in COVID-19 disease-related information was most evident during this period, when the suspected and confirmed cases of COVID-19 had already accumulated to 830 and 1072 respectively, scattered over 29 cities across China. Information exploded over the white-hot period: 1338 pieces of data were released over 18 days; with 57 pieces of disease-related, 5 pieces of public prevention-related, and 13 pieces of social welfare-related information were released per day.

The public's risk perception towards COVID-19 was dominated by "I do not know" (45\%) and "be alert, but not enough attention" (73\%) during the window period (Fig. 1, bar chart). Even at the rising period, 37\% of respondents still responded, "be alert, but not enough attention" and only half of them started to "take it seriously". Not until the white-hot period did the public start to pay attention to the epidemic. The public's responses of "take it seriously" and "fear and panic" rose to $80 \%$ and $4 \%$, while "be alert, but not enough attention" dropped to $13 \%$, yet still ranked as the top two most prevalent responses. A similar lag in the public's protective actions was also apparent (Fig. 2, bubble chart). Associated with the public's low-risk perceptions, over two- thirds of the respondents chose to "take no actions" during the window period, which was about 2-2.5 times higher than those starting to take "multiple actions" (25\%) (i.e. personal protection, self-isolation and family education). This ratio reversed in the rising period, where $65 \%$ of respondents took "multiple actions." and "take no actions" responses dropped to $24 \%$. By the white-hot period, respondents predominately took "multiple actions", increased by $61 \%$ as compared to the initial period.

\section{Discussion}

China, as the first country impacted by COVID-19, the success of containing this pandemic relies on the public's high trust in, support of and cooperation with the government [13]. Further, the success on controlling new incidence cases includes the collective willpower from the public built under the success risk communications. In reviewing the COVID-19 outbreak from the public's perspective, it is seen that insufficient information publicly available at the early stages of the outbreak made it more difficult to confine the virus with the least amount of costs. However, when faced with the new disease, China provided specific disease data to the $\mathrm{WHO}$ and the whole world for seeking more information and findings about COVID-19. As the WHO mentioned, although the initial stage is the window period in China, the publication of data and information is a contribution to the world, showing that the goal is to earn more time and more experiences on preventing the disease. Thus, our study highlights that timely release 
of information related to the epidemic, specific prevention instructions, and updates are imperative to prevent escalation of the epidemic.

Overall, our findings suggest the government should report the epidemic information logically and coherently, allowing the public, especially the elderly and other vulnerable populations, the time to be aware and to take appropriate protective actions as recommended. It is also noted that social media everincreasingly becomes an important channel to disseminate and reinforce information to the public, which should also be properly utilized to provide accurate, non- contradictory, and easy- to- understand messages. Multiple information channels combined with modern technology may facilitate the delivery of a visualized and timely message [14].

This work has been developed in an iterative process and references the body of work that addresses the effects of public risk communications to the individual's perceived risk and preventive actions. It is acknowledged that our survey sample recruited online may mainly present participants prone to internet information, as most Internet-based questionnaire studies $[15,16]$. While internet is ever-becoming the primary channel for rapid and timely dissemination and access to information [17, 18], our study provides vital evidence to understand people's trust of online information, thus to better guide their prevent behaviors via mass media. For the future study, the authors recommend more rounds of cognitive interviews or focus groups to ensure the survey items are accessible and understandable to a wide variety of individuals. Additionally, follow up interviews would flush out additional issues in the wording and structure of the survey. For the next step, it is necessary to confirm these relationships in the conceptual model through testing of the survey.

\section{Conclusion}

Our study identified when facing the pandemic, countries worldwide need to formulate prevention and control strategies tailored to their own situation, culture and traditions. WHO has detailed Risk Communication and Community Engagement strategies according to countries' outbreak stages $[19,20$, 21]: countries with no cases identified should communicate about preparedness measures and public health advice to manage uncertainty and misinformation; those with sporadic cases should establish and maintain trust with the public via ongoing two-way communication and provide guidance to encourage the adaptation of protective behaviors; while countries with clusters or community transmission should empower resilience in the public by ongoing risk communications and nimble support addressing people's concerns and needs. The cornerstone of these responses is the same for all countries, which is to engage the public's responsive actions. Every disease outbreak is an opportunity for us to learn about giving timely warning, optimizing public health advice, and providing frank communications. Weathering the global pandemic of COVID-19, our study urges countries worldwide to communicate level of risk and prevention strategies to the public authoritatively, clearly and timely. As firmly announced from the National Health Commission of the People's Republic of China and the WHO: being first, being right, and being credible $[14,22]$. 


\section{Abbreviations}

WHO: World Health Organization

COVID-19: the 2019 coronavirus epidemic

CCTV: China Central Television

\section{Declarations}

\section{-Ethics approval and consent to participate}

All data used were collected by the online survey via using Wenjuanxing Platform. All of participants have read and signed the informed context before starting the survey. Data were linked and analyzed only by authors who contribute writing the article.

-Ethics committeellnstitutional Review Board(IRB) of the Anthropology Department at Sun Yat-sen University, No: SYSUAD202003221.

-Consent for publication: Not applicable.

\section{-Availability of data and materials}

The data of survey results were conducted via the Wenjuanxing platform (https://www.wjx.cn/app/survey.aspx). More detailed data on participants' reactions in different periods cannot be provided because of confidentiality policies of Wenjuanxing, and Anthropology Department at Sun Yat-sen University.

\section{-Competing interests}

All authors declare no competing interests. The authors have completed the ICMJE United Competing Interest Form and declare no further competing interests.

\section{-Funding}

This study was funded by the emergency project of social science research under Yunnan University's first- class discipline of ethnology (B2018219). Funding body didn't give any interventions.

\section{-Author contributions}

JL, NG, MFZ, and DX contributed the idea for the review. JL and NG designed the study, conducted the review, and wrote the manuscript. XYJ and YDL collected primary data, analysis results and designed exhibits. MFZ, CYand DX contributed to the critical review of the manuscript. All authors reviewed and approved the final version. 
-Acknowledgments: We thank all the people who offered help for this study. We extend our deepest thanks to all of supports from School of Nursing Department, Sun Yat-sen University, Jinan University and Yunnan University.

\section{References}

1. World Health Organization. WHO Director- General's opening remarks at the media briefing on COVID19- 11 March 2020. Available online: https://www.who.int/dg/speeches/detail/who-director-generals-opening-remarks-at-the-media-briefing-on-covid-19-11-march-2020 (accessed on 11 March 2020).

2. Vinit Sharma D, De Beni. Annette Sachs Robertson. Why the Promotion of Family Planning Makes Sense now Than Ever Before? Available online: https://doi.org/10.1177/0972063420935545 (First published on August 5 2020).

3. World Health Organization. Coronavirus disease (COVID-19) advice for the public. Available online: https://www.who.int/emergencies/diseases/novel-coronavirus-2019/advice-for-public (Last updated 29 April 2020).

4. Cheng KK. Tai Hing Lam\& Chi Chiu Leung. Wearing face masks in the community during the COVID19 pandemic: altruism and solidarity. The Lancet. Published on April 162020.

5. Cable News Network (CNN) Politics. Trump declares national emergency- and denies responsibility for coronavirus testing failures. Available online: https://edition.cnn.com/2020/03/13/politics/donald-trump-emergency/index.html (accessed on 13 March 2020).

6. The Guardian Weekly. Italy's government orders all shops, bars and restaurants to close. Available online: https://www.theguardian.com/world/2020/mar/11/italy-faces-calls-to-close-everything-totackle-coronavirus (accessed on 12 March 2020).

7. DXY.DX Doctor. COVID-19 Global Pandemic Real-time Report. Available online: https://ncov.dxy.cn/ncovh5/view/pneumonia?from=dxy\&source=\&link=\&share=. (Updated on 30 April 2020).

8. World Health Organization. WHO Director- General's opening remarks at the media briefing on COVID19- 9 March 2020. Available online: https://www.who.int/dg/speeches/detail/who-director-general-sopening-remarks-at-the-media-briefing-on-covid-19--9-march-2020 (accessed on 9 March 2020).

9. Raymond Zhong. As Virus Spreads, Anger Floods Chinese Social Media. The New York Times. Available online: https://www.nytimes.com/2020/01/27/technology/china-coronavirus-censorshipsocial-media.html Published on 27 January 2020.

10. Zhongyun Big Data. https://zy.peopleyun.cn/index/index.do.

11. Kaiyuan, Sun. Jenny Chen \&Cecile Viboud. Early epidemiological analysis of the coronavirus disease 2019 outbreak based on crowdsourced data: a population - level observational study. Lancet Digital Health. 2020;2:e201-08. Published online. 20 February 2020. 
12. Jiang $H$, Jin L, Qian Xu. etc. Evidence of accessing antenatal care information via social media platforms supports mental wellbeing in COVID-19 epidemic. Bulletin of the World Health Organization. Submitted on 16 March 2020, Published online 18 March 2020.

13. National Health Commission of the People's Republic of China. Diagnosis andTreatment of New Coronavirus Pneumonia (Trial Version 6). Available online: http://www.nhc.gov.cn/jkj/s3578/202003/d29e176f35ad4b0a80c74c1d347bfbca.shtml (accessed on 7 March 2020).

14. European Center for Disease Prevention and Control. Outbreak of novel coronavirus disease 2019 (COVID-19): increased transmission globally- fifth update. Available online:https://www.ecdc.europa.eu/sites/default/files/documents/RRA-outbreak-novel-coronavirusdisease-2019-increase-transmission-globally-COVID-19.pdf (accessed on 2 March 2020).

15. Antunes B. Ben Bowers, etc. Anticipatory Prescribing in Community End-of-Life Care in the UK and Ireland During the COVID-19 Pandemic: Online Survey. BMJ Support Palliate Care. 2020. DOI:10.1136/bmjspcare-2020-002394.

16. Anta Gesser- Edelburg. Cohen R, Hijazi R. Nour Abed Elhadi Shahbari. Analysis of Public Perception of the Israeli Government's Early Emergency Instructions Regarding COVID-19: Online Survey Study. J Med Internet Res. 2020. DOI:10.2196/19370.

17. Priyanka Meel \& Dinesh Kumar Vishwakarma. Fake news, rumor, information pollution in social media and web: A contemporary survey of state-of-the-arts, challenges and opportunities. Experts Systems With Application. Accepted 26 September 2019. https://doi.org/10.1016/j.eswa.2019.112986.

18. Sharmistha Adhikari S, Ray M, Obaidat, Biswas GP. Efficient and secure content dissemination architecture for content centric network using ECG-based public key infrastructure. Computer Communications Revised 30 August 2019. https://doi.org/10.1016/j.comcom.2020.04.024.

19. World Health Organization. Report of the WHO-China Joint Mission on Coronavirus Disease 2019 (COVID-19). Available online: https://www.who.int/docs/default-source/coronaviruse/who-chinajoint-mission-on-covid-19-final-report.pdf (accessed on 16-24 February 2020).

20. World Health Organization. WHO Director- General's opening remarks at the media briefing on COVID19- 9 March 2020. Available online: https://www.who.int/dg/speeches/detail/who-director-general-sopening-remarks-at-the-media-briefing-on-covid-19--9-march-2020 (accessed on 9 March 2020).

21. World Health Organization. Risk communication and community engagement (RCCE) readiness and response to the 2019 novel coronavirus (2019-nCoV). Available online: https://www.who.int/publications-detail/risk-communication-and-community-engagement-readinessand-initial-response-for-novel-coronaviruses-(-ncov) (accessed on 26 January 2020).

22. Kai K. \& Jon Cohen. Can China's COVID-19 strategy work elsewhere? Science, March 6 2020, DOI: 10.1126/science.367.6482.1061.

\section{Figures}




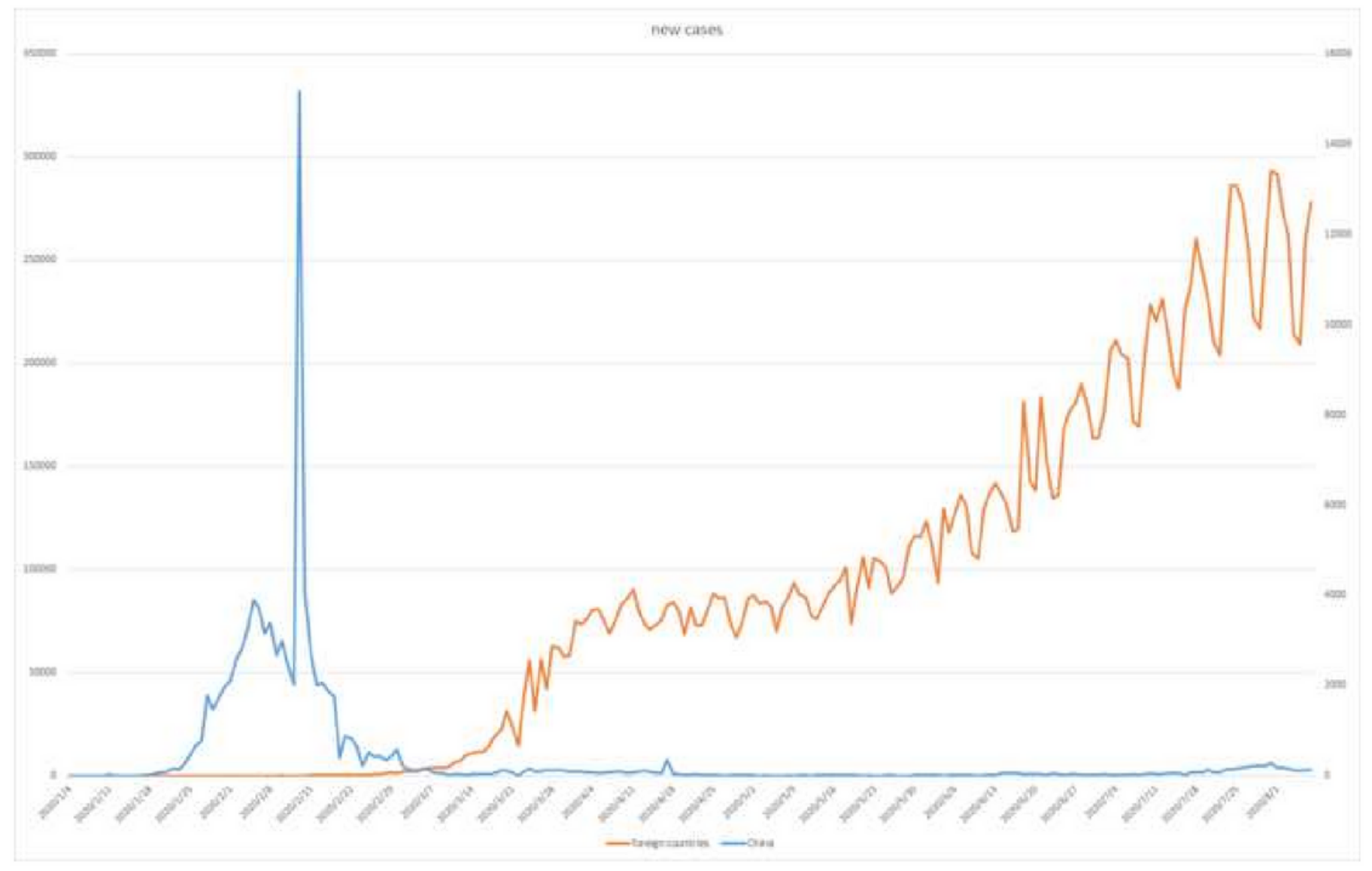

Figure 1

New cases reported weekly in China and other foreign countries 


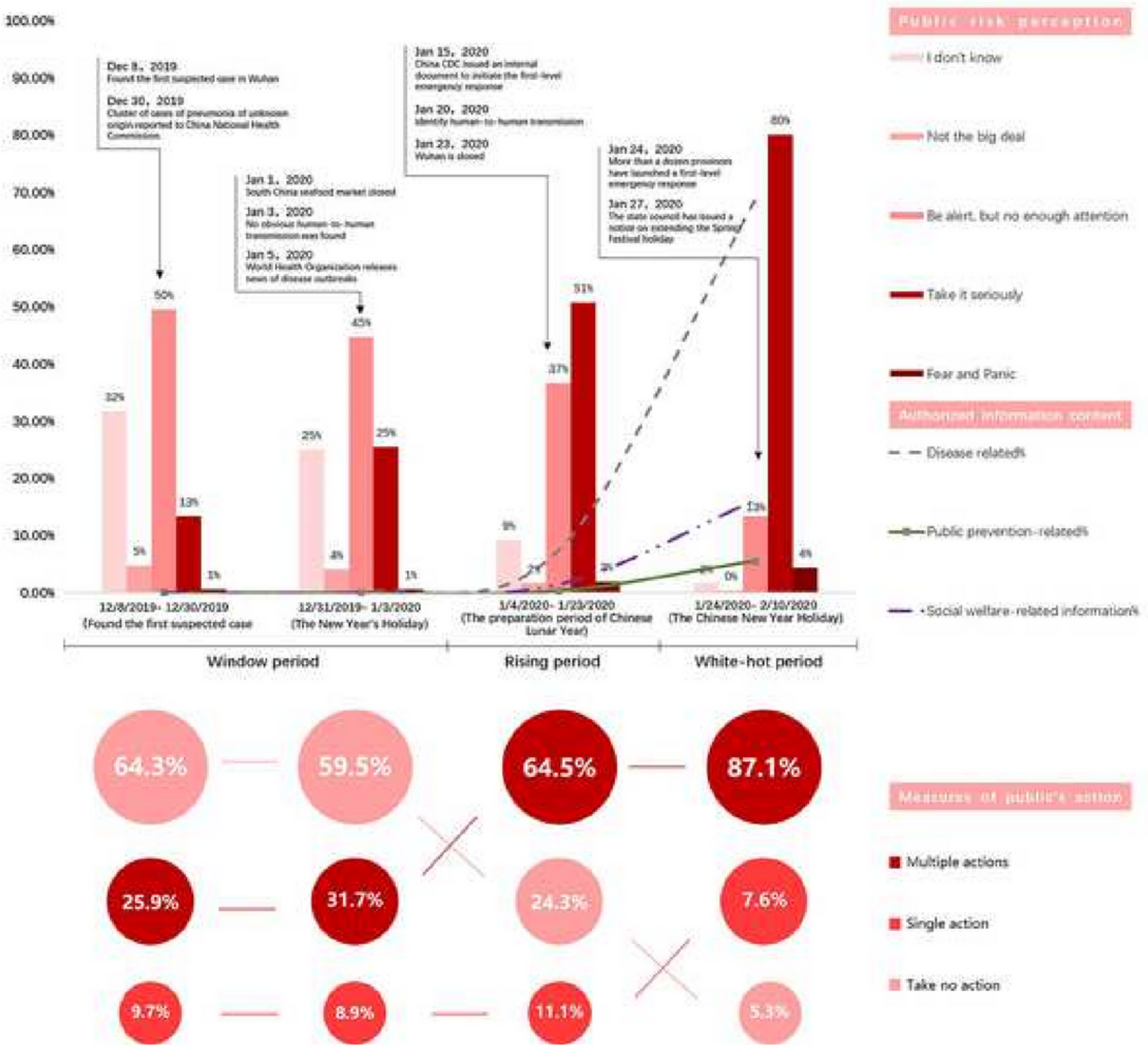

Figure 2

Type of authorized information provided, evaluated repeat measures of public risk perceptions, and actions towards COVID-19 over time (8 Dec 2019- 10 Feb 2020).

\section{Supplementary Files}

This is a list of supplementary files associated with this preprint. Click to download.

- Surveyquestions.docx 